\title{
Reciprocal Inhibition in Hemiplegia: Correlation with Clinical Features and Recovery
}

\author{
Yasuyuki Okuma and Robert G. Lee
}

\begin{abstract}
Background: Previous reports have described changes in reciprocal la inhibition in hemiplegic patients, but correlations between the amount of Ia inhibition and the clinical deficits have not been well established. Methods: We studied reciprocal inhibition between ankle flexors (tibialis anterior) and extensors (soleus) in 16 hemiplegic patients at various stages following a stroke and in 26 control subjects. The amount of disynaptic la inhibition was determined from the short latency suppression of the soleus or tibialis anterior H-reflexes by conditioning stimulation of the antagonistic muscle nerves. Results: Disynaptic Ia inhibition from peroneal nerve afferents to soleus motoneurones was increased in patients who showed good recovery of function with mild spasticity. However, it was not changed, or even sometimes diminished, in patients who made a poor recovery and had more marked extensor spasticity. In patients where serial recordings were obtained there was an increase in Ia inhibition during the recovery period following stroke. la inhibition to the tibialis anterior motoneurones tended to be greater in the poor recovery patients with marked spasticity than in the good recovery patients. The late (DI) inhibition, presumably due to presynaptic inhibition, was decreased in the patients, although consistent correlations between the amount of this inhibition and the clinical features were not clearly demonstrated. Conclusions: Changes in excitability of Ia inhibitory pathways can be correlated with some of the clinical features seen in hemiplegia. Increased la inhibition of soleus motoneurones during recovery may be a mechanism to compensate for loss of descending motor commands.
\end{abstract}

RÉSUMÉ: Inhibition réciproque dans l'hémiplégie: corrélation avec les manifestations cliniques et la récupération. Introduction: Des études antérieures ont décrit des changements dans l'inhibition réciproque la chez les patients hémiplégiques. Cependant. les corrélations entre le niveau d'inhibition la et les déficits cliniques n'ont pas été bien établies. Méthodes: Nous avons étudié l'inhibition réciproque entre les fléchisseurs de la cheville (jambier antérieur) et extenseurs (soléaire) chez 16 hémiplégiques à différents stades après un accident vasculaire cérébral et chez 26 contrôles. Nous avons déterminé le niveau d'inhibition disynaptique Ia au moyen de la suppression de la latence courte du réflexe $\mathrm{H}$ du soléaire ou du jambier antérieur par le stimulus conditionnel des nerfs des muscles antagonistes. Résultats: L’inhibition disynaptique la provenant des afférents du nerf sciatique poplité externe aux motoneurones du soléaire était augmentée chez les patients qui avaient une bonne récupération fonctionnelle avec spasticité légère. Cependant, elle était inchangée ou même parfois diminuée, chez les patients dont la récupération était médiocre et qui avaient une spasticité plus marquée au niveau des extenseurs. Chez les patients dont on a fait des enregistrements sériés, il y avait une augmentation de l'inhibition la pendant le phase de récupération après l'accident vasculaire cérébral. Le niveau d'inhibition la aux motoneurones du jambier antérieur avait tendance à être supérieur chez les patients dont la récupération était médiocre qui avaient une spasticité marquée par rapport à ceux dont la récupération était bonne. L'inhibition tardive (D1) était diminuée chez les patients. probablement par inhibition présynaptique, bien que nous n'ayons pas démontré clairement de corrélation entre le niveau de cette inhibition et les manifestations cliniques. Conclusions: Des changements de l'excitabilité des voies inhibitrices la peuvent être corrélés à certaines manifestations cliniques de l'hémiplégie. Une augmentation de l'inhibition la des motoneurones du soléaire pendant la récupération pourrait être un mécanisme compensatoire pour la perte de l'influx moteur.

Can. J. Neurol. Sci. 1996; 23: 15-23

Damage to the descending motor pathways in the brain results in contralateral hemiplegia. This is often accompanied by spasticity, weakness with a characteristic distribution, and other features of the upper motor neurone syndrome. Since some of these clinical features have been interpreted as examples of removal of tonic inhibitory influences, it may be useful to consider the changes in the activity of the spinal inhibitory interneurones as a consequence of interruptions of upper motoneurone pathways. ${ }^{\prime}$

The Ia inhibitory interneurone, which mediates reciprocal inhibition between agonist-antagonist muscle groups, has been studied extensively in animal experiments ${ }^{2}$ and in human subjects using $\mathrm{H}$-reflex techniques. ${ }^{3-12}$ Yanagisawa et al. ${ }^{5}$ reported

From the Department of Clinical Neurosciences, University of Calgary, Calgary. Dr. Okuma's present address is Department of Neurology, Juntendo University School of Medicine. Tokuo, Japan.

RECEIVED MAY 30, 1995. ACCEPTED IN FINAL FORM AL'GI'ST 23, 1995

Reprint requests to: Dr. R.G. Lee. Department of Clinical Neurosciences. Universily of Calgary, 3330 Hospital Drive N.W., Calgary, Albera. Canada T2N 4N1 
an imbalance in reciprocal Ia inhibition in hemiplegic patients. Inhibition of ankle flexor (dorsiflexor) motoneurones from afferents arising from ankle extensor (plantarflexor) muscles was strong, whereas Ia inhibition in the opposite direction from dorsiflexor afferents to extensor motoneurones - was very weak. They suggested that excessive Ia inhibition of flexor motoneurones might be one of the factors contributing to loss of function in ankle flexors. Evidence supporting this was provided by the observation that alcohol injections at the motor points of extensor muscles not only relieved spasticity but also improved strength in the ankle flexor muscles. ${ }^{5}$

The patients studied by Yanagisawa et al. were mainly ones with stable hemiplegic deficits and well established spasticity. It is possible that activity in reciprocal Ia inhibitory pathways may vary in relation to the type and severity of symptoms or the length of time since the stroke. Furthermore, the previous study on hemiplegic patients ${ }^{5}$ did not show results obtained from healthy control subjects studied under the same experimental conditions, so it is uncertain to what extent the abnormalities described are specific for hemiplegia.

For these reasons we examined reciprocal inhibition in hemiplegic patients at various stages of recovery following a stroke and attempted to establish correlations between patterns of reciprocal inhibition and clinical features, such as severity of weakness and degree of spasticity. In some patients we were able to examine the evolution in reciprocal Ia inhibition with serial recordings on two or more occasions following a stroke. The results indicate that there is considerable interindividual variation in reciprocal inhibition following a hemiplegic stroke and that there is some correlation with the clinical abnormalities.

\section{METHODS}

\section{Subjects and General Procedures}

We studied 16 patients with hemiplegia following a stroke (age 27-76 years, mean 57.1, S.D. 14.8) and 26 healthy control subjects (age 24-74 years, mean 51.6, S.D. 14.6). All subjects gave informed consent following a full explanation of the purpose of the research and the nature of the experimental procedures. The clinical data for the patients are summarized in the Table. They were all alert and cooperative. The interval between the onset of the stroke and testing ranged from two weeks to 10 years. Thirteen patients had a cerebral infarct involving the internal capsule or corona radiata. In three patients (no. 4, 14, 16) the stroke was due to an intracerebral hemorrhage in the basal ganglia region involving the posterior limb of the internal capsule. Strength for ankle dorsiflexion and plantarflexion was graded from 0 to 5 using the standard MRC scale, ${ }^{13}$ and strength of the tibialis anterior muscle was used as an indicator of functional recovery. Spasticity of the ankle extensor muscles was graded from 0 to 4 according to the Ashworth scale. ${ }^{14}$ None of the patients had contractures of the ankle joint. All patients had Babinski reflexes on the hemiplegic side and had marked weakness (less than grade 3 power on the MRC scale) in the tibialis anterior muscle immediately following the stroke. None were taking medication to relieve spasticity.

The experimental procedures were similar to those previously described..$^{12}$ Subjects were seated comfortably in a reclining arm chair with the feet resting on a pair of foot plates which kept both legs in a symmetrical posture with the angles of the knee and ankle joints at approximately 120 and $110 \mathrm{deg}$, respectively.

\section{Test Reflexes}

Excitability of the motoneurone pool was assessed by the size of the test $\mathrm{H}$-reflex responses recorded from the soleus and tibialis anterior muscles. Surface electrodes were used for both stimulation and recording. Paired electrodes were placed over the bellies of the soleus and tibialis anterior muscles. Interelectrode distance was $3 \mathrm{~cm}$. The tested muscles were in a relaxed state and EMG activity was monitored to ensure that no voluntary activation was occurring at the time of stimulation. The posterior tibial nerve was stimulated through a monopolar stimulating electrode (diameter $1 \mathrm{~cm}$ ) in the popliteal fossa to elicit $\mathrm{H}$-reflexes from the soleus muscle. The anode, $3 \mathrm{~cm} \times 2.5 \mathrm{~cm}$ metal plate, was placed over the anterior part of the patella. The test $\mathrm{H}$-reflexes in the tibialis anterior muscles were elicited by stimulating the common peroneal nerve at the level of the head of the fibula. The position of the cathode for peroneal nerve stimulation was adjusted to evoke selective contraction of the tibialis anterior, without activation of the peroneal muscles. The duration of the test stimulus was $1 \mathrm{~ms}$ for both the tibial nerve and the peroneal nerve. The reflex responses were amplified and stored on a computer disc with a sampling frequency of $5 \mathrm{kHz}$. Peak to peak amplitude was measured on line. At the beginning of each experiment, the amplitude of the maximum M-wave (M-max) was measured, and the size of the unconditioned test $\mathrm{H}$-reflexes was expressed as a percentage of M-max. Since it is well known that the same conditioning stimulus may have different effects on monosynaptic reflexes depending on the size of the test reflex, ${ }^{9,11}$ we kept the test H-reflex size between 20 and $40 \%$ of M-max in all the subjects when we tested the soleus $\mathrm{H}$ reflex. When we examined the tibialis anterior H-reflex, we kept the test size between 5 and $10 \%$ of M-max except for the initial recording from patient 1 ( $20 \%$ of M-max). Provided that the size principle of motoneurone recruitment applies to gradation of H-reflexes in the control and patient groups, we can assume that comparable populations of motor units were tested in both groups. ${ }^{12}$

\section{Conditioning Stimuli}

Conditioning stimuli were applied to the peroneal nerve for testing reciprocal inhibition of the soleus H-reflex or to the tibial nerve for inhibition of the tibialis anterior H-reflex, using the same electrodes used to elicit test H-reflexes. Single pulses of 1 ms duration were used for both peroneal and tibial nerve stimulation. The intensity of the conditioning stimulus was expressed in multiples of the motor threshold (xMT). For stimulation of the peroneal nerve, the intensity was always kept just at motor threshold $(1.0 \mathrm{xMT})$ for the tibialis anterior muscle. For conditioning stimuli to the tibial nerve, intensities less than the motor threshold for the soleus muscle were used because stronger stimuli often evoked a large soleus H-reflex with wide signal spread making it difficult to distinguish the tibialis anterior $\mathrm{H}$ reflex. For this reason, stimulus intensities between 0.75 and $0.83 \times$ MT were used in both control and patient groups for conditioning the tibialis anterior H-reflex. 
Table: Clinical Features and H-reflex Measurements in the Hemiplegic Patients.



\section{Footnotes for the table}

Inf. ; Infarction. Hem. ; Hemorrhage. IC ; Internal capsule. BG ; Basal ganglia.

ACA ; Anterior cerebral artery territory. MCA ; Middle cerebral artery territory.

Put ; Putamen. Thal ; Thalamus.

TA ; Tibialis anterior muscle. GS ; Gastrocnemius-soleus muscles.

MRC scale ; $5=$ normal strength, $4=$ enough to hold against moderate pressure, $3=$ enough to hold against gravity, $2=$ not enough to hold against gravity, $1=$ trace, $0=$ no contraction of muscle. Spa. ; Spasticity of the ankle extensor (plantarflexor) muscle determined by Ashworth scale $(0=$ no increase in tone, 1 = slight increase in tone, 2 = moderate increase in tone, $3=$ marked increase in tone, $4=$ severe spasticity, affected part rigid)

Clo.; Ankle clonus determined by clonus scale $(0=$ absence of clonus, $1=$ clonus 1 or 2 beats, $2=$ clonus more than 2 beats, but not sustained, $3=$ sustained clonus)

$\mathrm{H} / \mathrm{M}$; The ratio of maximum H-reflex to maximum M-wave.

$\mathrm{CPN} \rightarrow$ Soleus ; Reciprocal inhibition from common peroneal nerve (CPN) to Soleus H-reflex. PTN $\rightarrow$ TA ; Reciprocal inhibition from posterior tibial nerve (PTN) to TA H-reflex.

test- $\mathrm{H}$; The size of the test $\mathrm{H}$-reflexes expressed as a percentage of maximum $\mathrm{M}$-wave.

Ia.inh ; Amount of disynaptic la inhibition (\% of control value), D1.inh ; Amount of late (D1) inhibition (\% of control value).

NE ; Not examined. - ; H-reflex not elicited. \# ; Data from 4 subjects who showed measureable H-reflexes.

**:; $p<0.01 * ; p<0.05$

\section{Stimulus Protocol and Analysis of the Results}

The unconditioned and conditioned stimuli were presented randomly every 3 seconds. Eight to ten conditioned $\mathrm{H}$-reflexes and the same number of the unconditioned (control) H-reflexes were recorded for each conditioning-test (C-T) interval.
Reciprocal inhibition was demonstrated by plotting the amplitude of the conditioned $\mathrm{H}$-reflexes as a percentage of the unconditioned H-reflex, and the time course of this relationship was examined for C-T intervals ranging from 0.5 to $20 \mathrm{~ms}$. To examine reciprocal Ia inhibition of the tibialis anterior $\mathrm{H}$-reflex, $\mathrm{C}-\mathrm{T}$ 
intervals from $-2.0 \mathrm{~ms}$ (test stimulus preceding the conditioning stimulus by $2.0 \mathrm{~ms}$ ) to $+3.0 \mathrm{~ms}$ were used. Statistical comparisons were made using analysis of variance (ANOVA) or Student's $t$ test. For multiple comparisons, the Tukey method was used when ANOVA showed a significant difference. Probability values less than 0.05 were considered significant.

\section{RESULTS}

\section{H-reflexes in Control Subjects and Hemiplegic Patients}

The Table summarizes the data for the ratio of $\mathrm{H}$-max to $\mathrm{M}$ $\max (\mathrm{H} / \mathrm{M}$ ratio) in both soleus and tibialis anterior muscles. The soleus $\mathrm{H} / \mathrm{M}$ ratio in the patients ranged from 50 to $95.6 \%$ (mean 66.2, S.D. 13.6) and these values were significantly larger than the corresponding values for control subjects (mean 49.9 , S.D. 16.4) $(\mathrm{p}<0.01)$. There was no correlation between soleus $\mathrm{H} / \mathrm{M}$ ratio and degree of extensor spasticity. $\mathrm{H}$-reflexes from tibialis anterior muscles were elicited in only 6 out of 26 control subjects. In contrast, H-reflexes could be elicited from tibialis anterior in 15 out of 16 hemiplegic patients (H/M ratio ranged from 0 to $47.2 \%$ ). There was no correlation between the $\mathrm{H} / \mathrm{M}$ ratio and either the strength of tibialis anterior muscles or degree of extensor spasticity.

\section{Reciprocal Inhibition from Ankle Flexors to Extensor Motoneurones}

Figure 1-A illustrates the time course of the effects of peroneal nerve stimulation on the soleus $\mathrm{H}$-reflex in a normal control subject. There are two periods of inhibition. The first starts with a C-T interval of 0.5 to $1 \mathrm{~ms}$ and reaches a maximum at around 2 $\mathrm{ms}$. A later inhibition appears after $8 \mathrm{~ms}$ and lasts for more than 20 $\mathrm{ms}$. The timing of the short latency inhibition is compatible with disynaptic la inhibition which has been studied extensively in animal experiments. ${ }^{2}$ The later inhibition corresponds to what has been termed "DI inhibition" which is considered to represent presynaptic inhibition ${ }^{3.4}$ To estimate the amount of inhibition in an individual subject, the inhibitory effects were measured at the points of maximal depression of the H-reflex between 1.5 and 2.5 $\mathrm{ms}$ for disynaptic la inhibition and between 15 and $20 \mathrm{~ms}$ for the late (DI) inhibition as illustrated in Figure 1-B. The amount of Ia inhibition was small in the control subjects with a mean value of 7.1\% (S.D. 7.8\%) inhibition. Only one normal subject (illustrated in Figure 1) showed more than $20 \%$ inhibition. 15 subjects showed less than $10 \%$ inhibition.

Figure 2 shows the relationship between the amount of disynaptic Ia inhibition and the patients' clinical features. As shown in Figure 2-A, the amount of inhibition tended to increase as the spasticity decreased. A significant increase in Ia inhibition was found in the less spastic patients (degree of spasticity 0 or 1 ) (p $<0.001$ ). There was no significant difference between grade 2 or 3 patients and control values. In Figure 2-B, there was also a significant increase in Ia inhibition in the patients with strong tibialis anterior muscle strength (MRC scale 4 or 5) compared with control subjects $(\mathrm{p}<0.001)$.

In 4 patients (no. 1, 2,3,4) changes in reciprocal inhibition were observed with serial recordings during the recovery period following stroke. At the time of the initial study, muscle strength in the tibialis anterior muscle was weak and there was already some increase in soleus muscle tone in all these patients. The data from these 4 patients were included in Figure 2 and were

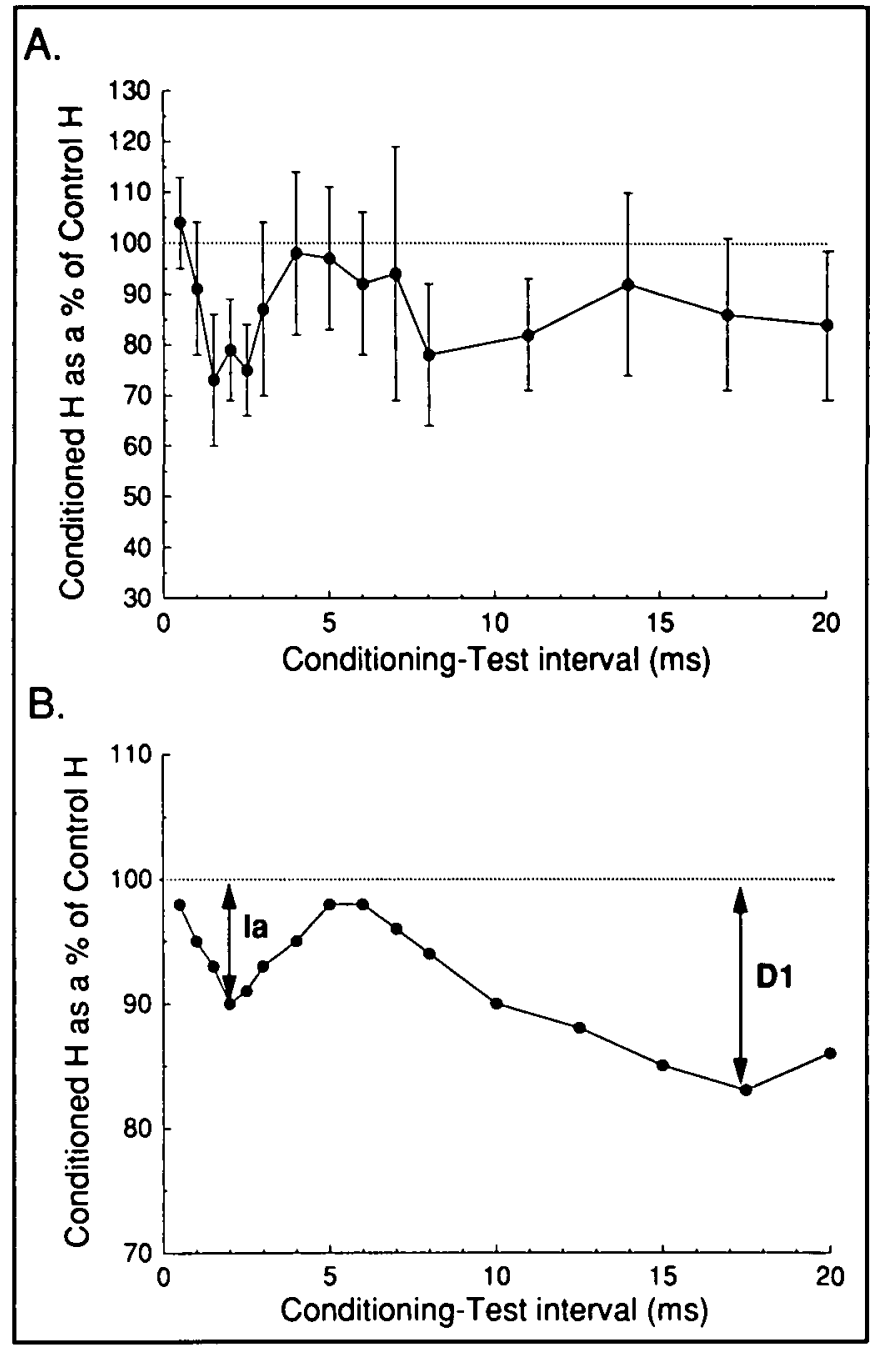

Figure 1: A: Time course of reciprocal inhibition of the soleus H-reflex by group I volleys from the peroneal nerve for a control subject. Values on the ordinate represent the amplitude of the conditioned $H$-reflex expressed as a percentage of the control (unconditioned) $H$-reflex amplitude. Each point is a mean of 8 observations with standard deviation. B: Schematic illustration showing how disynaptic la inhibition (Ia) and late inhibition (DI) were measured.

indicated with different symbols. In all these patients the amount of Ia inhibition increased as the strength of the tibialis anterior increased or as the degree of spasticity decreased. Patients 1 and 2 who had relatively mild spasticity showed a marked recovery of strength in ankle dorsiflexion (Table). Figure 3 illustrates the changes in the reciprocal inhibition in patient 1. A significant increase in the disynaptic la inhibition in the affected leg was observed in association with recovery of function (Figure 3-A, ANOVA: including data from 1.5 to 2.5 $\mathrm{ms}: \mathrm{P}<0.001)$. However, there was no significant change in the unaffected leg (Figure 3-B). The degree of spasticity remained mild in this patient. A significant increase in disynaptic la inhibition was also observed in patient $2(0 \%$ at the initial study, $17 \%$ in 4 months after stroke at a time of excellent recovery, see Table and data points in Figure 2 indicated with closed squares). In patients 3 and 4 , however, in whom spasticity did not resolve 


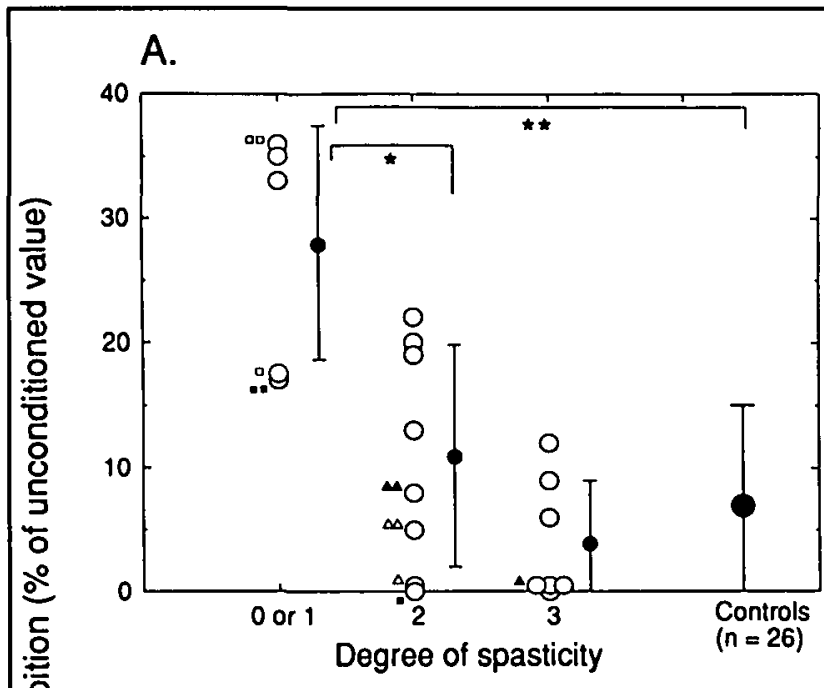

B.

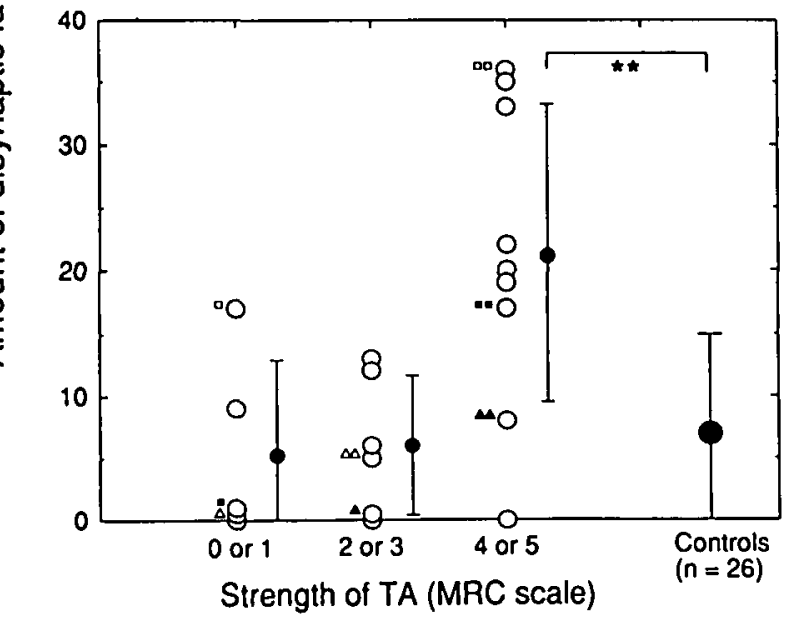

Figure 2: The relationship between disynaptic Ia inhibition and the clinical features in hemiplegic patients. The ordinates show the amount of inhibition determined as illustrated in Figure 1-B. The abscissa for A indicates the degree of spasticity. The abscissa for $B$ indicates the strength of the tibialis anterior muscle. Each open circle represents one subject, or one testing occasion when repeated examinations were performed. Small symbols beside the open circles indicate patients who were examined on more than one occasion. Single symbols indicate the initial examination; paired symbols the second examination. Open squares: patient I, closed squares: patient 2, open triangles: patient 3 , closed triangles: patient 4 (see Table). Data from the third examination in patients 1 and 2 were not included in this figure because there had been very little changes in strength or degree of spasticity from the second to the third examination. Closed circles and error bars indicate mean and one standard deviation.

$*_{*}^{*} ; p<0.001, * ; p<0.01$

well despite some recovery of muscle strength, the changes in the reciprocal inhibition were small.

Two patients (12 and 16) showed facilitation of the soleus $\mathrm{H}$ reflex. The latency of the facilitation was 3.0 and $2.5 \mathrm{~ms}$ respectively, and facilitation greater than $10 \%$ of the control value lasted for at least 3 consecutive C-T intervals. This type of facilitation in capsular hemiplegia has already been reported by Yanagisawa et al..$^{5}$ and was attributed to Ib facilitation. Since the onset of this facilitation occurs with C-T intervals from 2.5 to 3 $\mathrm{ms}^{5}$ and we measure Ia inhibition around $1.5-2.5 \mathrm{~ms}$, we would not expect to miss the important part of the Ia inhibition even if both effects were combined.

The late (D1) inhibition in the control subjects ranged from 5 to $35 \%$, with values mostly between 10 and $20 \%$, and a mean of $16.0 \%$ (S.D. 6.3\%). The mean amount of DI inhibition in the patients was $10.4 \%$ (S.D. $5.8 \%$ ), a value significantly smaller than in the controls ( $p<0.01$, Table). Figure 4 shows the relationship between the amount of late (DI) inhibition and the associated clinical features. The amount of D1 inhibition for each degree of spasticity tended to be smaller than in controls, but the differences were not significant, nor was there any significant difference between each subgroup in the patients. There was also no correlation between tibialis anterior muscle strength and the amount of D1 inhibition (Figure 4-B).

\section{Reciprocal Inhibition from Ankle Extensors to Flexor Motoneurones}

In four of 26 control subjects, it was possible to examine reciprocal Ia inhibition from the tibial nerve to the tibialis anterior H-reflex. Figure 5 illustrates the time course of this inhibition. It started when the test stimulus preceded the conditioning stimulus by $1 \mathrm{~ms}(\mathrm{C}-\mathrm{T}$ interval $=-1.0 \mathrm{~ms})$ and reached maximum when conditioning and test stimuli were delivered simultaneously (C-T interval $=0 \mathrm{~ms}$ ). A similar time course was obtained from the other three subjects. The maximum amount of inhibition in each subject ranged from $46 \%$ to $65 \%$ (mean $54 \%$ ) at a $\mathrm{C}-\mathrm{T}$ interval of around $0 \mathrm{~ms}$. It was obvious that the inhibition of the tibialis anterior H-reflex caused by tibial nerve stimulation was much stronger than that of the soleus $\mathrm{H}$-reflex by peroneal nerve stimulation.

In 8 patients (no. $1,3,6,11,12,13,14,16$ ) reciprocal la inhibition from the tibial nerve to the tibialis anterior motoneurones could be tested ([PTN $\rightarrow$ TA] in Table). The mean intensity of the conditioning stimulus was $0.79 \times \mathrm{xMT}$ (S.D. 0.04) and this value was not different from that in the control subjects $(0.78$ $\mathrm{xMT}$, S.D. 0.04). Figure 6 shows the relationship between the amount of disynaptic Ia inhibition and clinical features. The la inhibition tended to be larger as the degree of spasticity increased (Figure 6-A). On the other hand, inhibition tended to be greater in the patients with poor recovery in tibialis anterior muscle strength. These relationships showed trends which appeared to be the reverse of what was observed with inhibition of the soleus H-reflex by peroneal nerve stimulation.

\section{Discussion}

An important finding in the present study was a correlation between the amount of reciprocal la inhibition and the severity of the clinical abnormalities in hemiplegic patients. We found a significant increase in disynaptic reciprocal la inhibition from peroneal nerve to soleus motoneurones in patients who recovered good strength in the tibialis anterior muscle without developing marked spasticity in the ankle extensors. On the other hand, Ia inhibition remained weak, but with values similar to control subjects, in patients who had poor recovery of tibialis anterior muscle strength and more marked extensor spasticity. More direct evidence for this relationship was the increase in Ia inhibition in individual patients in whom repeated 


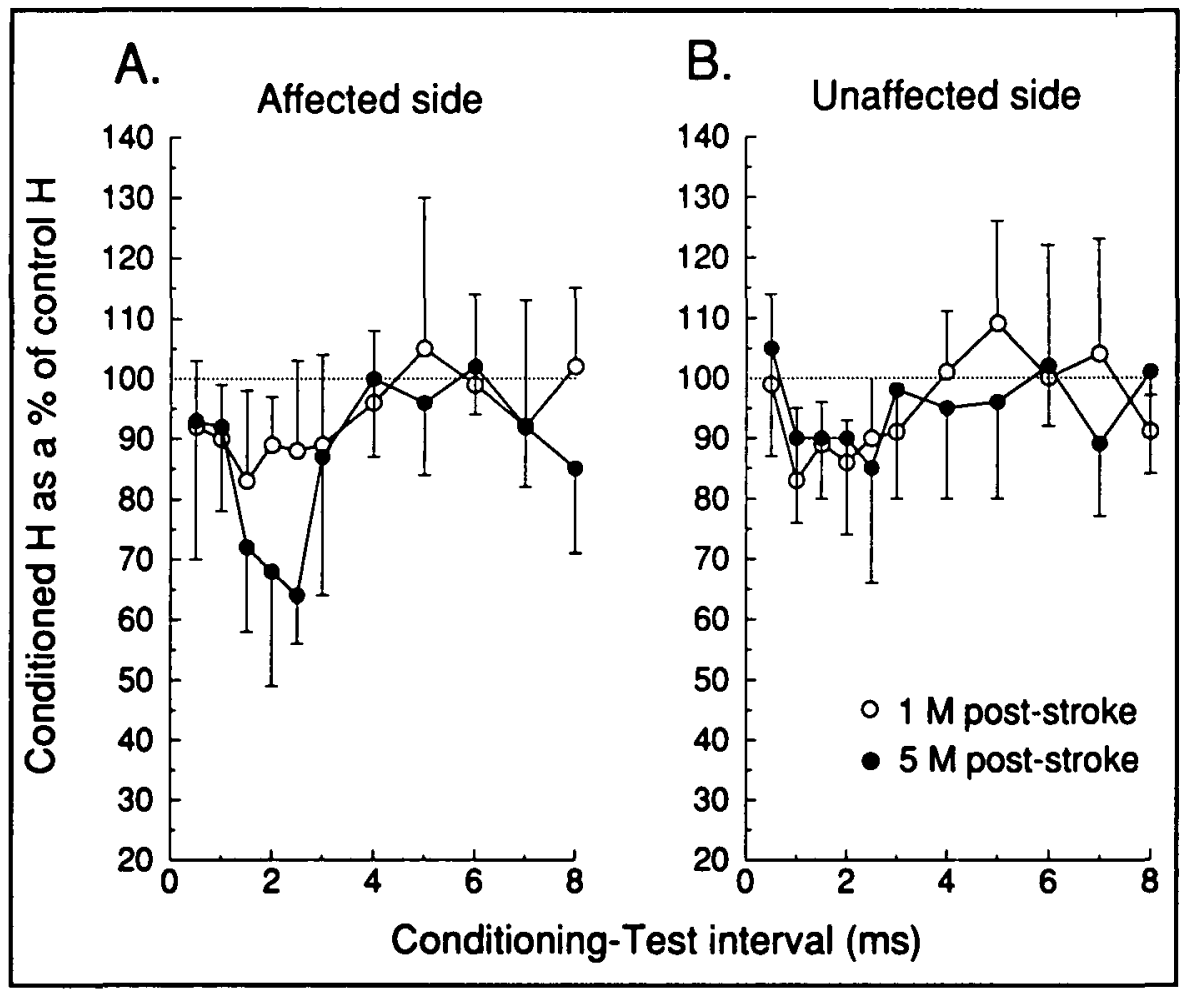

Figure 3: Changes in reciprocal Ia inhibition between I month (open circles) and 5 months (closed circles) post-stroke recordings in patient 1. Recordings were made from both legs at each experiment. A: Affected side. B: Unaffected side. The conventions are the same as in Figure 1-A.

recordings were obtained during the course of recovery from stroke.

\section{Comparison with Previous Studies}

Changes in reciprocal la inhibition in spasticity have been reported previously. Yanagisawa et al. ${ }^{5}$ studied 11 hemiplegic patients and found evidence of slightly increased Ia inhibition of soleus motoneurones in only two subjects. This could be partly due to the fact that in order to produce small M-waves to monitor stability of stimulations the stimulus intensity was such that the $\mathrm{H}$-reflexes were near maximum size. Later, Yanagisawa ${ }^{6}$ stated that half-maximum $\mathrm{H}$-reflexes were used in a few experiments but the results were essentially similar. Recently Boorman et al., ${ }^{12}$ using smaller test $\mathrm{H}$-reflexes $(20-40 \%$ of $\mathrm{M}$-max), reported increased Ia inhibition of the soleus motoneurones in the patients with spasticity due to incomplete spinal cord injury. They attributed the discrepancy between Yanagisawa's results (small Ia inhibition) and their own results (increased Ia inhibition) to the difference in the site of the lesion responsible for spasticity. However, the present study revealed that the Ia inhibition was actually increased in some hemiplegic stroke patients showing good functional recovery. Our results suggest that the amount of reciprocal inhibition is influenced by the severity of the clinical abnormalities. For instance, if we had examined only the severely affected patients with marked extensor spasticity, we might have concluded that Ia inhibition is not changed in hemiplegia.

In contrast to what was observed with the soleus H-reflex, we found a tendency for disynaptic Ia inhibition from tibial nerve to tibialis anterior motoneurones to be greater in the weaker and more spastic patients, and less in the good recovery group. Yanagisawa et al..$^{5}$ also reported strong inhibition of tibialis anterior motoneurones in three patients, but a relationship with the clinical features was not established. Ashby and Wiens, ${ }^{15}$ using peri-stimulus time histograms (PSTHs), demonstrated increased Ia inhibition of ankle dorsiflexor motoneurones in patients with spinal spasticity. They examined the firing probability of motor units in the tibialis anterior muscle, and constructed PSTHs, to show the effects of tibial nerve stimulation. However, since their observations were made during voluntary contraction, it is not possible to compare these results directly to our findings.

\section{Functional Implications}

Figure 7 summarizes the results and possible functional consequences for the changes which we have observed. In control subjects (Figure 7-A) Ia inhibition from ankle dorsiflexor (tibialis anterior) afferents to extensor (soleus) motoneurones is weak (indicated by a thin dotted line), whereas inhibition to the flexor motoneurones is strong (thick line). This quantitative asymmetry of reciprocal inhibition in normal subjects has been reported previously. 4.10

In the patients who have poor recovery and marked spasticity (Figure 7-B), the asymmetry of Ia inhibitory effects between ankle dorsiflexors and extensors is the same as in normal controls, or even more marked. The "release mechanism" indicated in Figure 7-B and $\mathrm{C}$ is intended to illustrate a reduction of descending tonic inhibitory influences which are present under normal conditions. This would effectively result in a "release" of facilitation. The strong "Ia" inhibition from extensor afferents 


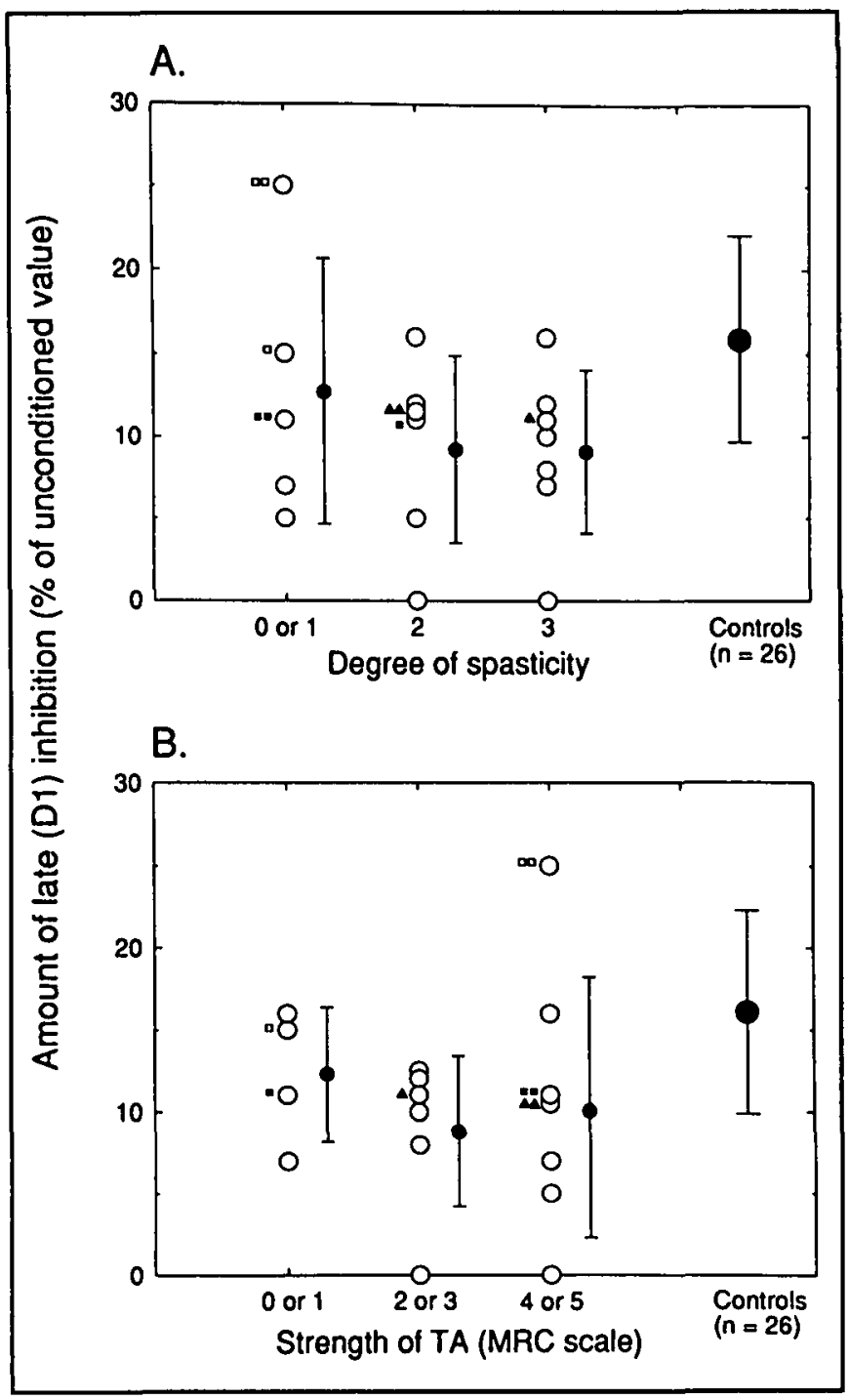

Figure 4: The relationship between the late $(D 1)$ inhibition and clinical features in the hemiplegic patients. The conventions used for axes and symbols are the same as in Figure 2.

to flexor systems (thick line) may cancel a large part of these release effects on the flexors. ${ }^{5}$ Thus the tibialis anterior motoneurones cannot become sufficiently excitable to compensate for the severe loss of descending motor drive. As a result, ankle dorsiflexion remains weak.

In the good recovery patients with less spasticity (Figure 7-C), increased activity of the Ia inhibitory pathway from flexor afferents to extensor motoneurones is indicated by thick hatched line towards the soleus $\alpha$-motoneurone. This increased excitability in the flexor Ia inhibitory interneurones may improve the situation for the patients with reduced descending motor drive by facilitating recruitment of inhibition of the antagonist muscles, something which is indispensable for smooth voluntary movements. Reciprocal inhibition is particularly important at movement onset when a dynamic stretch of antagonist muscles may initiate clonus in individuals with spasticity. Previous studies ${ }^{16,17}$ have shown

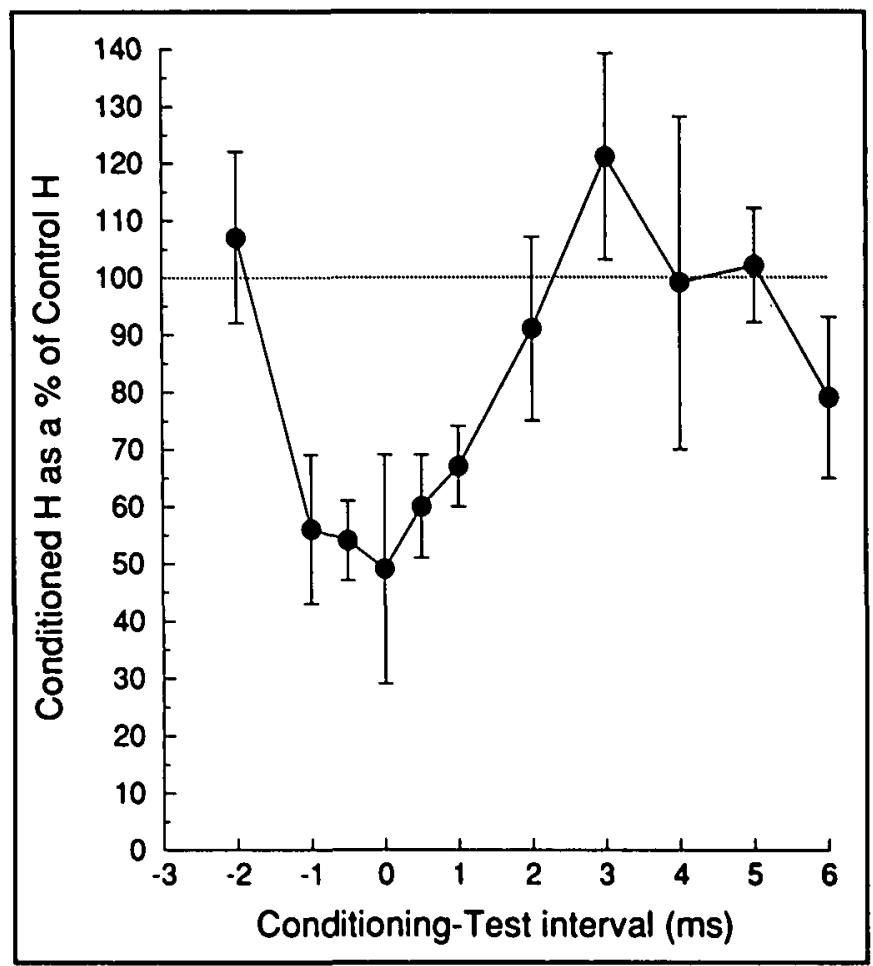

Figure 5: Time course of reciprocal inhibition of the tibialis anterior $H$-reflex by group I volleys from the tibial nerve for a control subject. The conventions are the same as in Figure I-A.

appropriately timed activation of reciprocal inhibition at this stage.

Another important finding was the presence of $\mathrm{H}$-reflexes in the tibialis anterior muscles in almost all of the patients. Since a tibialis anterior $\mathrm{H}$-reflex could not be elicited in most control subjects, this may be interpreted as evidence for increased excitability in the tibialis anterior motoneurones due to the release mechanism. The increased excitability of the tibialis anterior motoneurones may compensate to some extent for a reduced descending motor drive. ${ }^{5}$ Thus, in the patients with good recovery, increased activity of both Ia interneurones and $\alpha$ motoneurones for the ankle flexors would contribute to well regulated voluntary ankle dorsiflexion movements.

\section{Possible Mechanisms for Changes in Hemiplegic Patients}

The exact mechanisms for the release effect are not fully understood, but if they are due to reduction of tonic inhibitory influences from supraspinal centers,' then it might be suspected that these effects have a stronger influence on extensor muscles in hemiplegics since spasticity is usually present in these muscles but not in the flexors. The strong la inhibition from the extensor afferents to the flexors in the more spastic patients (thick line in Figure 7-B) could be the result of stronger release effects. One of the possible consequences of the release mechanisms would be excessive Ia discharges from muscle spindles in the spastic soleus, secondary to gamma hyperactivity as suggested by Yanagisawa et al. ${ }^{5}$ The increased la afferent activity may excite extensor Ia inhibitory interneurones and inhibit flexor Ia interneurones through mutual inhibitory connections. Thus Ia 


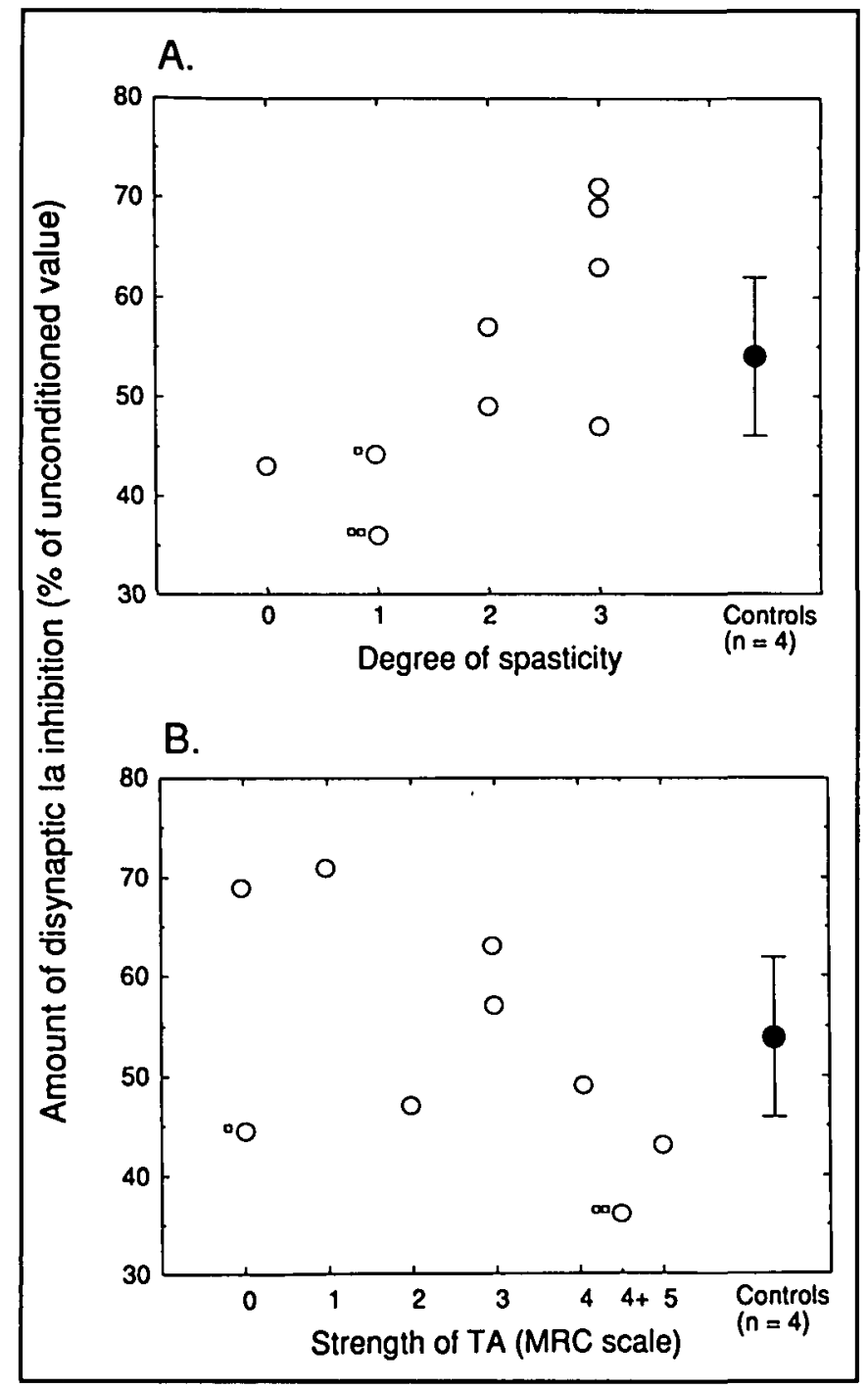

Figure 6: The relationship between the la inhibition of the tibialis anterior $H$-reflex and clinical features. The conventions are the same as in Figure 2.

inhibition from flexors to extensor motoneurones would remain weak in this group. Excessive Ia discharge in spastic patients has not been confirmed by microneurographic recordings, probably because of the technical difficulties, and further studies of this type are needed. ${ }^{18}$

What might account for increased Ia inhibition from the flexor afferents to the extensor motoneurones in the patients with good recovery? It is possible that in some patients intensive physiotherapy and repeated attempts to produce ankle dorsiflexion movements, one of the most difficult tasks for hemiplegics, could, over a long term, modify synaptic transmission in the Ia inhibitory pathway to the antagonist extensors because Ia inhibition to the extensors becomes active during voluntary ankle dorsiflexion. ${ }^{4,8}$ The mechanisms may be similar in some respects to what Yanagisawa ${ }^{6}$ proposed to explain the exaggerated la inhibition observed in the adult type of athetotic cerebral palsy. ${ }^{3} \mathrm{He}$ suggested that the ability to produce consistent localized reciprocal inhibition during voluntary movement may be a compensatory mechanism acquired by these patients as a result of prolonged exercise programs designed to generate normal functional movements. The increased Ia inhibition in our hemiplegic patients could be partially due to passive release mechanisms as illustrated in Figure 7, but an active mechanism similar to that proposed for the cerebral palsy patients could be an important contributing factor as well.

\section{Late (D1) Inhibition}

We also observed a decrease in the late (D1) inhibition in our hemiplegic patients. This inhibition was first described by Mizuno et al., ${ }^{3}$ and because of its long time course and low effective threshold,,$^{3,4}$ it was attributed to presynaptic inhibition of Ia afferents. Therefore it might be suspected that presynaptic inhibition was decreased in some of the hemiplegic patients, but since we could not demonstrate a clear correlation between the amount of Dl inhibition and the degree of spasticity, it cannot. be concluded from our results that decreased presynaptic inhibition is a major factor responsible for the spasticity. The concept that decreased presynaptic inhibition contributes to spasticity has been supported by observations of decreased vibratory inhibition of the soleus H-reflex. ${ }^{19}$ However, human experiments using continuous vibration should be interpreted cautiously, because vibration can activate Golgi tendon organs and can also spread to the antagonist muscle spindles. ${ }^{20}$ These factors may cause postsynaptic effects on test $\mathrm{H}$-reflexes which cannot be distinguished from the effects of presynaptic inhibition. Hultborn et al. ${ }^{21}$ have developed a novel method to assess changes in presynaptic inhibition by examining heteronymous facilitation from quadriceps to soleus motoneurones. In a recent study $^{22}$ this method was applied to evaluate presynaptic inhibition in patients with spasticity.

In summary, we have presented evidence to show that excitability of Ia inhibitory systems undergoes a number of changes during the recovery period following stroke. In patients who have good functional recovery there is increased Ia inhibition from ankle dorsiflexors to extensor (plantarflexor) motoneurones. This might contribute to the recovery by serving as a compensatory mechanism for the reduced descending motor commands to flexor Ia interneurones. In patients with poor recovery and marked spasticity in the soleus this compensatory mechanism would not be effective. In contrast, Ia inhibition from extensors to flexor motoneurones showed a reverse trend with greater Ia inhibition being associated with poor recovery and marked extensor spasticity. This strong Ia inhibition would suppress the activity of both flexor (tibialis anterior) alpha motoneurones and the corresponding Ia interneurones. As a result there would be persisting weakness of the tibialis anterior and reduced reciprocal inhibition of the soleus. 


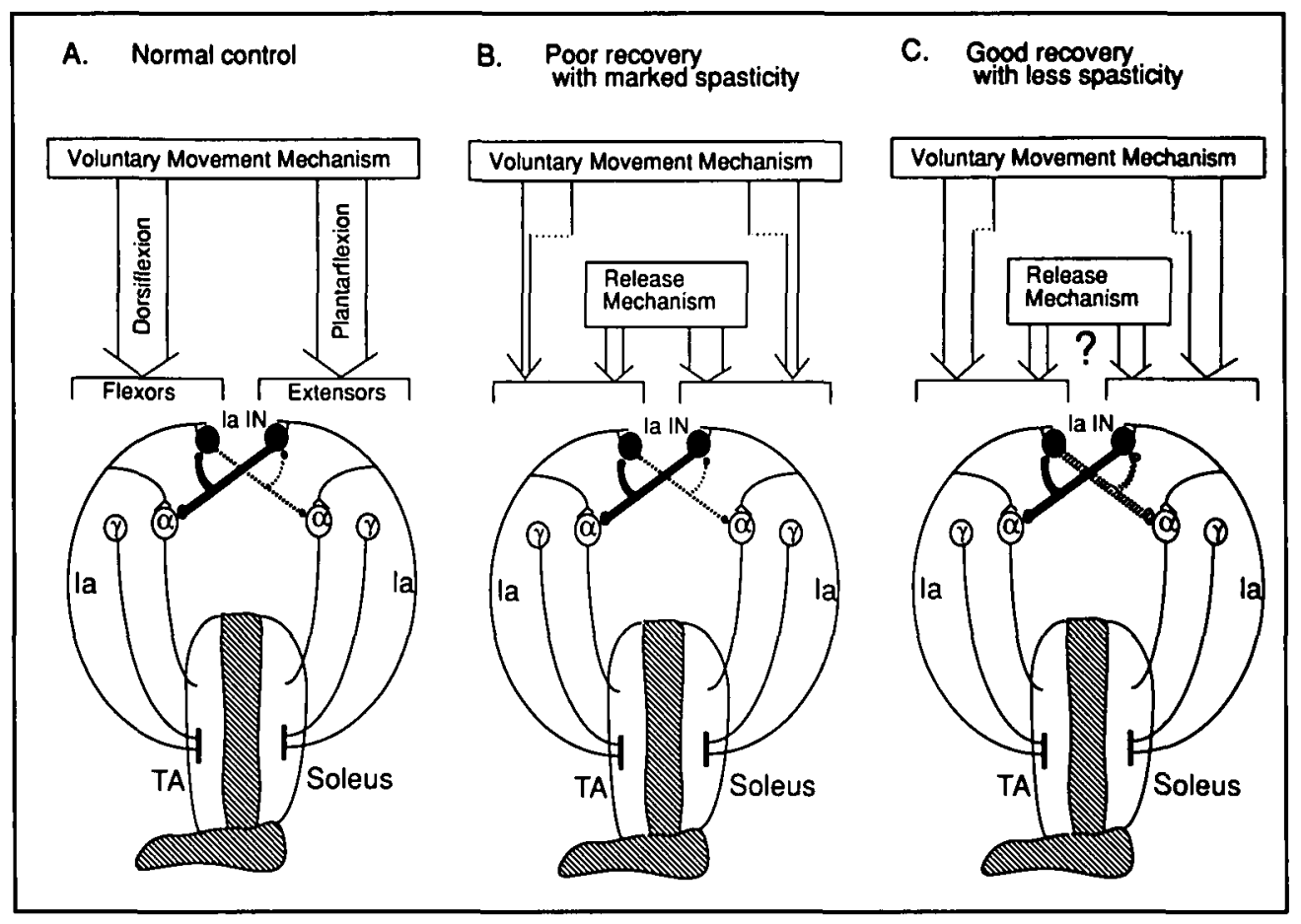

Figure 7: Schematic illustration of neural networks between ankle dorsiflexor (TA) and extensor (soleus) muscles. $\alpha$ : alpha-motoneurones. $\gamma:$ gammumotoneurones. Ia: la afferent fibers. Neurones shown as filled circles indicate la inhibitory interneurones (Ia IN). They have mutual inhibitory connections (indicated by small curved line). Thicker lines from the cell bodies indicate higher level of excitability of the la inhibitory interneurones. The short bars in the muscles indicate muscle spindles.

\section{ACKNOWLEDGEMENTS}

We thank Dr. R. Tanaka, Professor N. Yanagisawa, and Dr. M Shindo for their valuable suggestions and critical reading of the manuscript. We are also grateful to Ms. I. S. O'Callaghan and Dr. K. Oi for their assistance

\section{REFERENCES}

1. Lee RG, Boorman G. Upper motoneurone syndrome: clinical aspects and treatment. In: Berardelli A, Benecke R, Manfredi M, Marsden CD, eds. Motor Disturbances II. Academic press, 1990: 333-345.

2. Baldissera $F$, Hultborn $H$, Illert $M$. Integration in spinal neuronal systems. In: Brooks VB, ed. Handbook of Physiology. section 1 , The Nervous System, Vol.2, Motor Control. Bethesda: American Physiological Society, 1981: 509-595.

3. Mizuno $Y$, Tanaka $R$, Yanagisawa $N$. Reciprocal group I inhibition on triceps surae motoneurones in man. J Neurophysiol 1971; 34: 1010-1017.

4. Tanaka R. Reciprocal la inhibition during voluntary movements in man. Exp Brain Res 1974; 21: 529-540.

5. Yanagisawa N, Tanaka $R$, Ito $Z$. Reciprocal Ia inhibition in spastic hemiplegia of man. Brain 1976; 99: 555-574.

6. Yanagisawa $\mathrm{N}$. Reciprocal reflex connections in motor disorders in man. In: Desmedt JE, ed. Progress in Clinical Neurophysiology, vol. 8, Spinal and Supraspinal Mechanisms of Voluntary Motor Control and Locomotion. Basel: Kargar, 1980, 129-141.

7. Pierrot-Deseilligny E, Morin C, Bergego C, Tancov N. Pattern of group I fiber connections from ankle flexor and extensor muscles in man. Exp Brain Res 1981; 42: 337-350.

8. Shindo M, Harayama H, Kondo K, Yanagisawa N, Tanaka R. Changes in reciprocal la inhibition during voluntary contraction in man. Exp Brain Res 1984; 53: 400-408.

9. Crone C, Hultborn H, Jespersen B. Reciprocal la inhibition from the peroneal nerve to soleus motoneurones with special reference to the size of the test reflex. Exp Brain Res 1985; 59: 418-422.

10. Crone C, Hultborn H, Jespersen B, Nielsen J. Reciprocal Ia inhibition between ankle flexors and extensors in man. J Physiol 1987; 389: $163-185$
11. Crone C, Hultborn H, Mazieres L, et al. Sensitivity of monosynaptic test reflexes to facilitation and inhibition as a function of the test reflex size: a study in man and the cat. Exp Brain Res 1990; 81: $35-45$.

12. Boorman G, Hulliger M, Lee RG, Tako K, Tanaka R. Reciprocal la inhibition in patients with spinal spasticity. Neurosci Lett 1991; 127:57-60.

13. Medical Research Council. Aids to the examination of the peripheral nervous system. Memorandum No.45 London: HMSO, 1976, 1-2.

14. Ashworth B. Preliminary trial of carisoprodol in multiple sclerosis. Practitioner 1964; 192; 540-542.

15. Ashby $P$, Wiens $M$. Reciprocal inhibition following lesions of the spinal cord in man. J Physiol 1989; 414: 145-157.

16. Kagamihara $Y$, Tanaka $R$. Reciprocal inhibition upon initiation of voluntary movement. Neurosci Lett 1985; 55: 23-27.

17. Tanaka R. Spinal cord circuits in man - with a special reference to the reciprocal Ia inhibitory pathway. In: Ellingson RJ, Murray NMF, Halliday AM, eds. The London symposia (EEG Suppl 39): Elsevier, 1987; 67-71.

18. Hagbarth KE. Microneurography and applications to issues of motor control: Fifth annual Stuart Reiner memorial lecture. Muscle \& Nerve 1993; 16: 693-705.

19. Delwaide PJ. Human monosynaptic reflexes and presynaptic inhibition. An interpretation of spastic hyperreflexia. I $n$ : Desmedt JE ed. New Developments in Electromyography and Clinical Neurophysiology, vol.3, Human Reflexes, Pathophysiology of Motor systems, Methodology of Human Reflexes. Basel: Karger, 1973; 508-522.

20. Burke D, Hagbarth KE, Lofstedt L, Wallin G. The responses of human muscle spindle endings to vibration of non-contracting muscles. J Physiol 1976; 261: 673-693.

21. Hultborn H, Meunier S, Morin C, Pierrot-Deseilligny E. Assessing changes in presynaptic inhibition of la fibers: a study in man and the cat. J Physiol 1987; 389: 729-756.

22. Faist M, Mazevet D, Dietz V, Pierrot-Deseilligny E. A quantitative assessment of presynaptic inhibition of la afferents in spastics. Differences in hemiplegics and paraplegics. Brain 1994; 117 : 1449-1455. 